\title{
Los sistemas acuapónicos como fuente de alimento con la implementación de nuevas tecnologías
}

Importance of aquaponic systems as a food source with the implementation of new technologies

\author{
Mario Andrés Colorado Gómez ${ }^{1}$ \\ Magnolia Herrera Marchena ${ }^{2}$ \\ Jordán Andrés Lancheros González ${ }^{3}$ \\ Fundación Clínica Shaio \\ Centro de Microscopía y Microanálisis (CEMMI)
}

Iván Darío López Buitrago ${ }^{4}$ Ricardo Antonio Buitrago Bernal ${ }^{5}$ Jorge Reynolds Pombo ${ }^{6}$ Fundación Clínica Shaio

\footnotetext{
${ }^{1}$ mariocolorado@hotmail.com https:/orcid.org/0000-0002-0333-4896

2 magnolia.herrera@shaio.com https://orcid.org/0000-0002-5664-1362

3jordan.lancheros@shaio.com https://orcid.org/0000-0002-5618-7084

${ }^{4}$ ivan.lopez@shaio.com https://orcid.org/0000-0002-9308-5255

${ }^{5}$ ricardo.buitrago@shaio.com http://orcid.org/0000-0002-2602-5455

6 jorgereynolds@hotmail.com https://orcid.org/0000-0002-5490-697 


\section{Resumen}

Para el desarrollo de cualquier ser vivo es indispensable el consumo u obtención de nutrientes del medio en el que se encuentra; en consecuencia, si los seres vivos (plantas, animales, hongos, etc.) se emplean en sistemas productivos, el tema de los nutrientes cobra aún más importancia pues ya no solo se busca que el ser vivo sobreviva, sino que crezca de una manera óptima. El principio de la acuaponía se basa en la integración de la acuicultura (producción de especies acuáticas) con la hidroponía (cultivo de plantas en ausencia de suelo) mediante el empleo de sistemas de recirculación de agua y nutrientes. En el esquema general del modelo acuapónico, el alimento que se le suministra a los peces funciona como la entrada de energía al sistema, de donde obtienen los nutrientes necesarios para crecer y vivir. Como cualquier organismo, los peces asimilan del alimento lo necesario y los compuestos que obtienen en exceso junto con aquellos que son producto o desecho de su metabolismo, los excretan. Estos desechos y el alimento no consumido por los peces se convierten en el sustrato del que las bacterias, hongos y microalgas, toman los nutrientes necesarios para aumentar su población. Esta sinergia, permite la transformación de residuos tóxicos para el mismo sistema acuático como el nitrito de amonio, en nitratos; moléculas que las plantas aprovechan para desarrollarse, crecer, producir biomasa y frutos ricos en nutrientes, objetivo primordial de un sistema de producción de plantas. Sin embargo, estos procesos de transformación de la energía requieren del control de los parámetros físicos y químicos que permitan el bienestar de todos los seres vivos que convergen en el sistema de producción acuapónica. En consecuencia, ha sido muy importante validar el funcionamiento de un sistema de monitoreo remoto en tiempo real de los parámetros de calidad del agua, y las variables ambientales a través de la implementación de sistemas de comunicación apoyados en el principio del Internet de las Cosas (IoT), el cual consiste en dotar a objetos con sensores y dispositivos, para que estos queden conectados a Internet a través de redes fijas e inalámbricas, permitiendo la recolección e intercambio de datos para su monitoreo, análisis y gestión en tiempo real y de esta forma se pueda tener control y seguimiento de lo que acontece dentro del sistema acuapónico.

Palabras clave: Acuaponia, Bacterias nitrificantes, IoT, Diseño. 


\section{Abstract}

For the development of any living being, it is essential to consume or obtain nutrients from the environment in which it is found; Consequently, if living beings (plants, animals, fungi, etc.) are used in productive systems, the issue of nutrients becomes even more important since it is not only sought that the living being survives, but also that it grows in an optimal way. The principle of aquaponics is based on the integration of aquaculture (production of aquatic species) with hydroponics (cultivation of plants in the absence of soil) through the use of water and nutrient recirculation systems. In the general scheme of the aquaponic model, the food that is supplied to the fish functions as the energy input to the system, from where they obtain the necessary nutrients to grow and live. Like any organism, fish assimilate what is necessary from food and the compounds that they obtain in excess together with those that are the product or waste of their metabolism, they excrete. These wastes and the food not consumed by the fish, become the substrate from which bacteria, fungi and microalgae take the necessary nutrients to increase their population. This synergy allows the transformation of toxic waste for the same aquatic system as ammonium nitrite, into nitrates; molecules that plants take advantage of to develop, grow, produce biomass and fruits rich in nutrients, the primary objective of a plant production system. However, these energy transformation processes require the control of physical and chemical parameters that allow the well-being of all living beings that converge in the aquaponic production system. Consequently, it has been very important to validate the operation of a remote monitoring system in real time of water quality parameters and environmental variables through the implementation of communication systems supported by the principle of the Internet of Things ( IoT), which consists of equipping objects with sensors and devices, so that they remain connected to the Internet through fixed and wireless networks, allowing the collection and exchange of data for monitoring, analysis and management in real time and of this way you can have control and monitoring of what happens within the aquaponic system.

Keywords: Aquaponics, nitrifying bacteria, IoT, Design. 


\section{Introducción}

La acuaponía puede definirse como un cultivo de peces y plantas en un sistema de recirculación cerrado, en un ambiente controlado. En términos generales es un sistema donde los desechos orgánicos producidos por los peces son convertidos, a través de la acción bacteriana, en nitratos, que sirven como fuente de alimento para plantas. Estas a su vez al tomar estos nitratos, limpian el agua para los peces donde actúan como filtro biológico (Nelson, 2007; Parker, 2002; Van Gorder, 2000) En este sistema, los nutrientes que excretan directamente los organismos cultivados en la producción acuícola (peces, camarones, bivalvos) o que son generados por la descomposición microbiana de los desechos orgánicos, son absorbidos y utilizados como nutrientes por las plantas cultivadas hidropónicamente (Roosta \& Hamidpour, 2011). Es así como este modelo sirve para una producción sostenible de alimentos, de acuerdo con los principios de reutilización de aguas residuales, la integración de sistemas acuícola-agrícola en un policultivo que incrementa la diversidad y producción final, y la posibilidad de obtener productos "más sanos" con importantes impactos socioeconómicos a nivel local (Diver, 2006; Colorado, 2019).

Es importante destacar que para el manejo del sistema acuapónico se deben tener en cuenta parámetros de calidad del agua como son: : el oxígeno disuelto, la temperatura, la conductividad eléctrica, los sólidos disueltos totales, los compuestos nitrogenados, los fosfatos, el $\mathrm{pH}$, la alcalinidad, la dureza, el dióxido de carbono, el calcio y el potasio (Rakocy et al., 2004; Lieth \& Oki, 2008; Rakocy, 2010), para permitir un balance entre los rangos óptimos de los parámetros calidad de agua de cada uno de los diferentes tipos de organismos cultivados (los peces, las plantas y las bacterias nitrificantes). El manejo de la calidad del agua y su disponibilidad constante son importantes, debido a que estos factores pueden ser determinantes de la producción de vegetales de consumo humano (Borges-Gómez et al., 2010). Otro factor importante a tener en cuenta es la dependencia de la energía la cual influye tanto en la aireación como en el flujo de agua ya que las plantas realizan respiración celular y pueden agotar rápidamente el oxígeno disuelto en el agua, generando a su vez alteraciones en el pH. (Malcom, 2005). Esto es muy importante, ya que una interrupción del bombeo por 
períodos cortos puede llevar a que el sistema colapse por lo que es necesario contar con plantas eléctricas, bombas de gasolina entre otras. Comercialmente, los sistemas acuapónicos están en sus inicios, pero como la tecnología se desarrolla y es redefinida cada día, estos sistemas tienen el gran potencial de ser un método más eficiente y sostenible para el cultivo de peces y vegetales (Adler et al., 2012) en acuaponía el desecho de los peces funciona como una fuente de alimento para las plantas y éstas a su vez, actúan como un filtro natural del agua en la que viven los peces (Somerville et al., 2014). Esto crea un mini ecosistema en donde tanto las plantas como los peces pueden vivir y prosperar (Colorado, 2019). La acuaponía es una alternativa ideal para solucionar el problema de los acuicultores para deshacerse del agua cargada de nitrógeno y, asimismo, contribuir a la solución del problema de los agricultores de cómo conseguir el nitrógeno para sus plantas (Mateus, 2009).

En este proyecto, se desarrolló un sistema acuapónico implementado con tecnología IoT, en el cual se monitorea y visualiza diferentes variables como lo son: la temperatura, la luz, el caudal de agua empleado, el grado de acidez del agua y la generación del color e intensidad de luces LED RGB, esto con el fin de establecer el avance que brinda esta tecnología para el desarrollo de un cultivo acuapónico. Teniendo en cuenta los avances tecnológicos, el cual nos permite estar conectados en cualquier instante y lugar; nace una tecnología que adaptamos a este proyecto llamada "Internet of Things", en donde implementamos dispositivos electrónicos como los sensores y el microcontrolador, para medir diferentes variables

\section{Diseño del sistema de recirculación acuapónico}

El sistema consta de los siguientes elementos: Sistema de acuarios, clarificador, camas de crecimiento para plantas, sistemas de bombeo de agua y sistemas de aireación. Estos elementos se conectan de tal forma que el agua rica en nutrientes pasa del tanque de peces al clarificador, donde se eliminan la mayor parte de partículas disueltas, tanto grandes como pequeñas. Otra posible función del clarificador es servir como receptáculo para la adición de elementos, del clarificador se pasa al biofiltro (figura 1). 
Figura 1. Sistema acuapónico

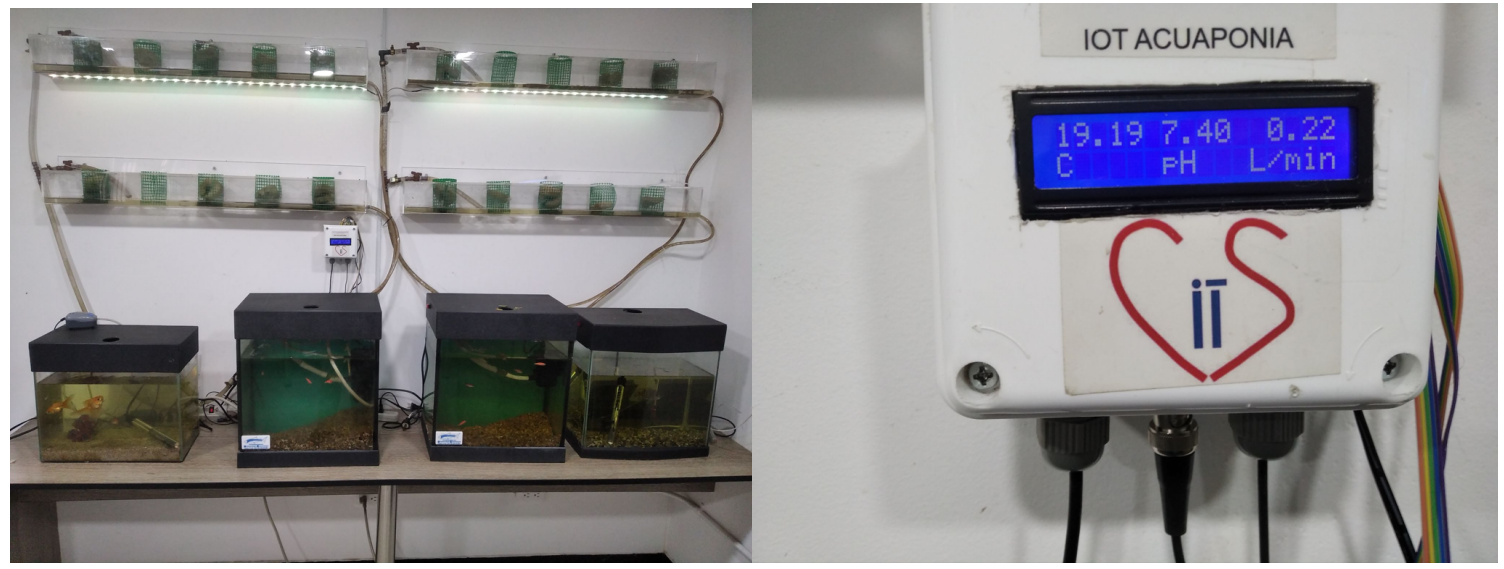

La tecnología implementada en el sistema acuapónico consta de sensores que interactúen con un módulo acuapónico, para procesar y mostrar en un display local las variables, además de permitir interacción a través de un pequeño servidor WEB, para que los usuarios puedan controlar la intensidad independientemente de cada fuente de luz.

\section{Desarrollo}

\subsection{Hardware y software libres}

Dado que el hardware tiene asociados a él costos variables directos, ninguna definición de software libre se puede aplicar directamente sin modificación. En cambio, el término hardware libre se ha usado principalmente para reflejar el uso del software libre con el hardware y el lanzamiento libre de la información con respecto al hardware, a menudo incluyendo el lanzamiento de los diagramas esquemáticos, diseños, tamaños y otra información acerca del hardware. De todos modos, incluye el diseño del hardware y la distribución de los elementos en la tarjeta madre. 


\subsection{Microcontrolador}

El cual fue empleado para realizar todas las tareas de medición, control y comunicaciones fue el ESP8266: Este constituye un Sistema en el Chip avanzado que nos brinda la conectividad necesaria y los dispositivos periféricos para realizar dichas tareas.

\subsection{Sensores utilizados para adquirir las variables físicas se enumeran a continuación}

. Sonda para medir el pH que determina el grado de acidez o alcalinidad del agua utilizada en el sistema acuapónico.

- Medidor de flujo de agua para determinar el caudal de agua empleado con el medidor de caudal YF-S201.

. Sonda con funda de acero que contiene el sensor de estado sólido DS18B20 para medir la temperatura del agua.

- Control de luces LED RGB, se realizó con un servidor WEB instalado en el microcontrolador ESP8266, programación WEB utilizando JavaScript para ajuste gráfico de las luces LED RGB mediante modulación del ancho del pulso PWM.

. Reloj de tiempo real para ser usado en la programación de la ejecución de tareas periódicas. El chip empleado para realizar dicha tarea es el DS1307.

- La pantalla LCD local utilizada para mostrar los datos, está basada en el controlador de hardware HD44780. Como bus de comunicaciones digitales se empleó la interfaz de comunicaciones I2C PCF8574 con un potenciómetro para el contraste de la pantalla.

- Fuente de alimentación del sistema utilizada para alimentar eléctricamente a todos los subsistemas, utiliza el regulador variable LM2596, ajustado a 5 voltios en la salida del 
circuito, y una fuente conmutada de 12 voltios que provee la alimentación eléctrica primaria de todo el sistema.

. Entorno de desarrollo $\mathbf{C}++$ para el software: Para la realización del programa se empleó el ambiente interactivo de desarrollo Arduino (IDE), debido a la gran variedad de librerías para desarrollo de software y a su facilidad de uso.

Figura 2. Sistema IoT para medición de variables físicas y control de luces RGB.

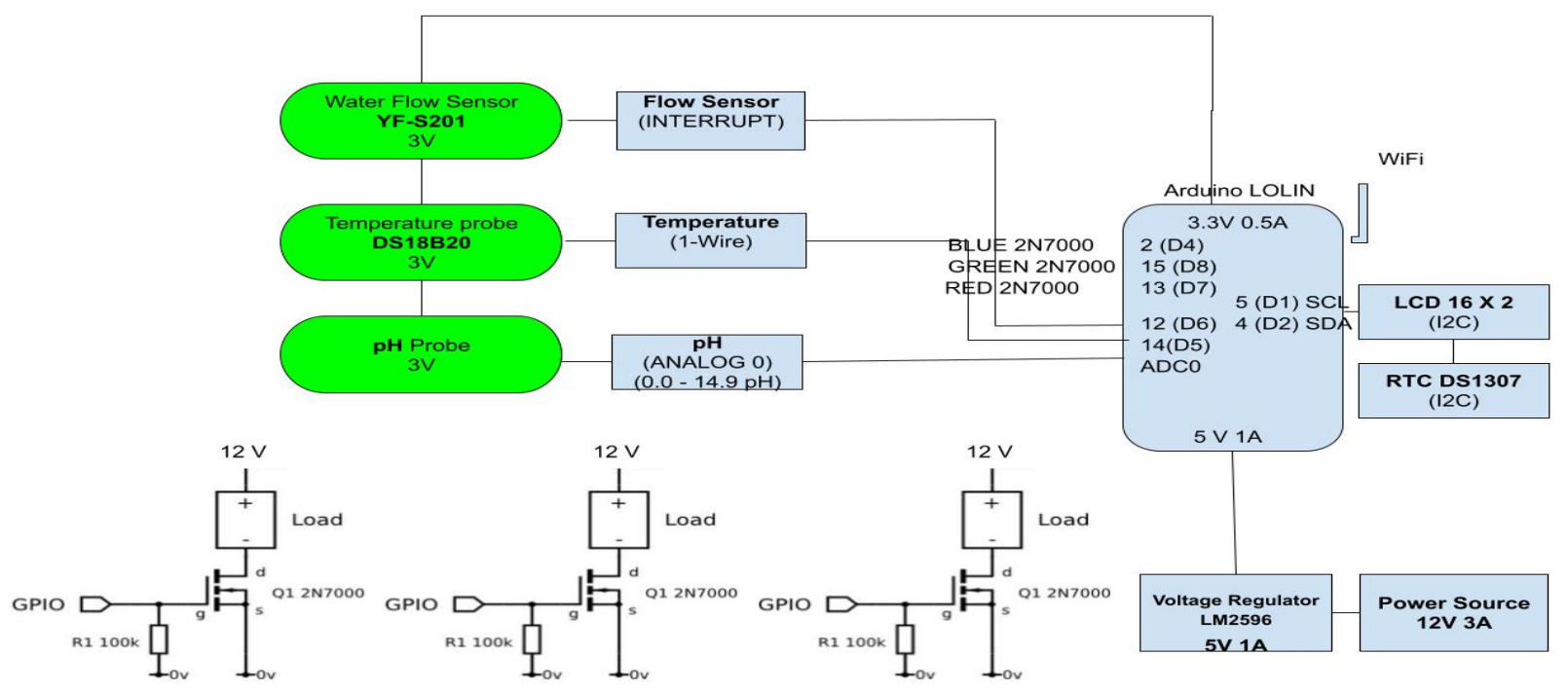

\subsection{Iluminación LED implementada}

El sistema busca la radiación fotosintética activa. Las primeras observaciones realizadas al sistema se realizaron en plantulas ubicadas con las raíces inmersas en el agua, usando una esponja como soporte para el cuerpo. Se realizaron pruebas de estimulación fotosintética con los diodos ubicados dentro de un rango del espectro de 400 a $450 \mathrm{~nm}$ y una distancia relativa entre las hojas y el LED de alrededor de 20 centímetros, donde se observa poco aprovechamiento de las plántulas de dicha longitud de onda y mueren al poco tiempo de "sembradas" presentando un aspecto seco en sus hojas a pesar de tener un flujo constante de agua (Badamasi, 2014). 
En una segunda etapa se iniciaron pruebas con led de luz cálida (amarilla) en dos tipos de montaje: Uno con sustrato y otro sin él. El sustrato utilizado en tierra de jardín con un capilar en el que se colocaron plántulas de 2 especies diferentes: lenteja y garbanzo. El otro montaje fue con un diente de ajo sostenido por la espuma, aunque no directamente tocando está el agua sino una parte de la raíz del diente.

En un periodo de 3 semanas se evidencia que el LED de luz cálida da mejor resultado que el de Azul en las plántulas de lenteja y garbanzo que en las lechugas en otros lados.

Se cambió la tonalidad de color a un naranja en la combinación de leds dado por el manejo del ESP8266 y se encontró una mejora en la duración de las plántulas ubicadas en agua.

Las que se encontraban en tierra se evidenciaban de crecimiento, pero su tallo es muy delgado y carece de fortaleza. El ajo durante más de tres semanas genera empieza de cero y alcanza a generar un tallo verde de casi $10 \mathrm{~cm}$ de largo.

\section{Conclusiones}

- La implementación de este tipo de sistemas de producción en Colombia tiene un alto potencial de desarrollo e importantes ventajas, en comparación con otros países. El clima, los recursos hídricos de fácil acceso, la amplia variedad de especies acuáticas y vegetales y la implementación de nuevas tecnologías, hacen de los sistemas acuapónicos una herramienta real y eficaz para obtener beneficios económicos y sociales en el país.

. Los sistemas de recirculación acuapónicos son un medio eficaz para reducir y aprovechar los residuos que normalmente son vertidos al ambiente. • Debido a que las plantas recuperan un porcentaje sustancial de los nutrientes disueltos, la tasa de intercambio de agua se puede disminuir. Esto reduce los costos de operación en los sistemas acuapónicos en los climas áridos y los invernaderos con calefacción donde el agua representa un gasto importante. 
. La implementación de nuevas tecnologías como lo es el IoT ayuda a facilitar las revisiones constantes del comportamiento de las variables fisicoquímicas, en especial el $\mathrm{pH}$ y los compuestos nitrogenados presentes en el agua, Ya que el tener controladas dichas variables nos asegura tener una buena producción tanto de plantas como de peces.

- Actualmente se están revisando otro tipo de luces para el crecimiento de las plantas en interiores con una combinación de led azul, rojo y blanco que permita el crecimiento y la fotosíntesis adecuada.

\section{Referencias}

Adler, P., R.; Takeda, F., Glenn, D., M.; Summerfelt, S., T. (2012). Utilizing byproducts to enhance aquaculture sustainability.

Badamasi, Y. A. (2014). The working principle of an Arduino. In 2014 11th international conference on electronics, computer and computation (ICECCO) (pp. 1-4). IEEE.

Borges-Gómez, L., Cervantes Cárdenas, L., Ruiz Novelo, J., Soria-Fregoso, M., Reyes Oregel, V. y Couoh, V. (2010). Capsaicinoids in Habanero pepper (Capsicum chinense Jacq.) under different conditions of moisture and nutrition. Terra Latinoamericana. 28 (1), 35 - 41.

Colorado Gómez, M. A. (2019). Monitoreo acuapónico y tecnología IOT en el CBA. Revista Siembra CBA, (1), 83-88.

Diver S. (2006). Aquaponics - Integration of Hydroponics with Aquaculture. National Sustainable Agriculture Information Service (ATTRA). 28p.

Lieth, J.H., Oki, L.R. (2008). Irrigation in Soilless Production. In: Raviv, M. y Lieth, J.H. (ed.). Soilless Culture: Theory and Practice. London, UK. Elsevier. 
Malcolm J. (2005). Backyard aquaponics. A guide to building an aquaponic system. Joel Malcolm, Western Australia.

Mateus, J. Red Hidroponía, Boletín №44. (2009). Lima-Perú. Acuaponía: hidroponía y acuacultura, sistema integrado de producción de ALIMENTOS, pp 7-10. http://www.lamolina.edu.pe/hidroponia/redhidro/boletin44/44_acuaponia.pdf

Nelson RL. (2007). Acuaponía. Nelson/Pade Multimedia.Montillo, WI. USA.

Parker R. (2002). Aquaculture science. Second edition. Delmar. Albany, NY. USA.

Rakocy JE, Shultz RC, Bailey DS, Thoman ES. (2004). Aquaponic production of tilapia and basil: comparing a batch and staggered cropping system. Acta Horticulturae (ISHS), 648:6369. http://www.actahort.org/books/648/648_8.htm

Rakocy, J.E. (2010). Aquaponics: integrating fish anbd plant culture. In: Timmons, M. y Ebeling, J. (ed.). Recirculating Aquaculture. 2nd Ed. Ithaca, NY, USA. Cayuga Aqua Ventures.

Roostaa HR, Hamidpour M. (2011). Effects of foliar application of some macro- and micronutrients on tomato plants in aquaponic and hydroponic systems. Scientia Horticulturae 129: 396-402.

Somerville, C., Cohen, M., Pantanella, E., Stankus, A. y Lovatelli, A. (2014). Producción de alimentos acuaponicos a pequeña escala: cultivo integrado de peces y plantas. Documento técnico de la FAO sobre pesca y acuicultura, (589).

Van Gorder SD. (2000). Small scale aquaculture. The Alternative Aquaculture Association. Breinigsville, PA, USA. 
REVISTA INTERNACIONAL DE PEDAGOGÍA E INNOVACIÓN EDUCATIVA

Volumen 2. Número 1. Enero - Junio 2022

ISSN: 2745-0341 (En línea) 